is even $\left(2^{a_{0}}\right)$ then the subgroup must involve operators of order 4 and $\alpha_{0}>3$. Since any number of these factors may be nonabelian, there cannot be an upper limit to the number of non-abelian groups which may be conformal with one abelian group. This fact may be seen in many other ways.

STANFORD UNIVERSITY, October, 1901.

\title{
THE INFINITESIMAL GENERATORS OF CERTAIN PARAMETER GROUPS.
}

BY DR. S. E. SLOCUM.

(Read before the American Mathematical Society, October 26, 1901.)

By means of the $r$ independent infinitesimal transformations

$$
X_{j} \equiv \sum_{1}^{n} \xi_{j k}\left(x_{1}, \cdots, x_{n}\right) \frac{\partial}{\partial x_{k}} \quad(j=1,2, \cdots, r)
$$

we may construct a family of transformations

$$
x_{i}^{\prime}=f_{i}\left(x_{1}, \cdots, x_{n}, a_{1}, \cdots, a_{r}\right) \quad(i=1,2, \cdots, n)
$$

with $r$ essential parameters $a_{1}, \cdots, a_{r}$, where $f_{i}(x, a)$ is defined in the neighborhood of the identical transformation by the series

$$
\begin{gathered}
f_{i}(x, a) \equiv x_{i}+\sum_{1}^{r} a_{j} X_{j} x_{i}+\frac{1}{2 !} \sum_{1}^{r} \sum_{i}^{r} X_{j} X_{i} x_{i}+\cdots \\
(i=1,2, \cdots, n) .
\end{gathered}
$$

The transformations defined by these equations for assigned values of the $a$ 's may be denoted by $T_{a}$. Let the differential operators $X_{j}(j=1,2, \cdots, r)$ satisfy Lie's criterion, that is, let

$$
X_{j} X_{k}-X_{k} X_{j} \equiv \sum_{1}^{r} c_{j k s} X_{s} \quad(j, k=1,2, \cdots, r) .
$$

Then by Lie's chief theorem, the family of transformations $T_{a}$, defined by equations (1), forms a group $G . *$ Conse-

\footnotetext{
* Continuierliche Gruppen, pp. 390-391.
} 
quently the transformation obtained by the successive application to the manifold $x_{1}, \cdots, x_{n}$, in the order named, of the transformations denoted by $T_{a}$ and $T_{b}$ respectively, with parameters $a$ and $b$, will be a transformation of the group $G$, say $T_{0}$; that is to say, we shall have

where

$$
T_{b} T_{a}=T_{c}
$$

$$
c_{k}=\varphi_{l k}\left(a_{1}, \cdots, a_{r}, b_{1}, \cdots, b_{r}\right) \quad(k=1,2, \cdots, r) .
$$

If this system of equations is written in the form

$$
a_{k}^{\prime}=\varphi_{k}\left(a_{1}, \cdots, \alpha_{r}, \alpha_{1}, \cdots, \alpha_{r}\right) \quad(k=1,2, \cdots, r),
$$

it can be shown that they define an $r$-pararneter group in the variables $a$ and $\alpha^{\prime}$, with continuous parameters $\alpha_{1}, \cdots, \alpha_{r}$, and also that each transformation of the group is generated by an infinitesimal transformation of the group. The group thus defined is termed the parameter group of the given group G.*

On pages 97-103 of the Proceedings of the American Academy of Arts and Sciences, volume 36, I have shown that the symbols of the infinitesimal transformations which generate the parameter groups are the same for all groups of the same structure ; and in the same pages I have also given a method by which these symbols may be obtained from the structural constants belonging to any given structure. The following is a résumé of the method given in that paper.

Let $\alpha$ denote the differential operator $\alpha=\sum_{1}^{r} a_{k} X_{k}$. Then equations (1) may be written in the symbolic form

$$
x_{i}^{\prime}=e^{a} x_{i} \quad(i=1,2, \cdots, n),
$$

where $e^{a}$ denotes the operator

$$
e^{\alpha} f=f+\alpha f+\frac{\alpha^{2}}{2 !} f+\frac{a^{3}}{3 !} f+\cdots
$$

and $\alpha^{m+1} f=\alpha\left(\alpha^{m} f\right)$. By making the parameters in equations (1) infinitesimal we obtain an infinitesimal transformation of the family, that is to say, a transformation infinitely near the identical transformation. Let $\delta t$ denote an infinitesimal, and let $\gamma$ denote the operator $\gamma=\sum_{i}^{r} c_{k} X_{k}$, the

\footnotetext{
* Transformationsgruppen, vol. 1, pp. 401 et seq.
} 
$c$ 's being arbitrary parameters. Then the transformation $e^{a+\delta t \gamma}$ is infinitely near the transformation $e^{\alpha}$. Consequently the transformation obtained by the successive application to the manifold $x_{1}, \cdots, x_{n}$, in the order named, of the transformation $e^{-\alpha}$, inverse to $e^{\alpha}$, and the transformation $e^{\alpha+\delta t \gamma}$ is an infinitesimal transformation. If we denote its parameters by $\delta t b_{1}, \cdots, \delta t b_{r}$ and let $\beta=\sum_{i}^{r} b_{k} X_{k}$, we have

$$
e^{-a} e^{a+\delta t \gamma}=e^{\delta t \beta}
$$

whence

$$
\begin{gathered}
1+\delta t\left\{\gamma-\frac{1}{2 !}(\alpha, \gamma)+\frac{1}{3 !}[\alpha,(\alpha, \gamma)]-\frac{1}{4 !}\{\alpha,[\alpha,(\alpha, \gamma)]\}+\cdots\right\} \\
+\cdots \cdots=1+\delta t \beta+\cdots,
\end{gathered}
$$

where $(\alpha, \gamma)$ denotes the alternant $\alpha \gamma-\gamma^{\alpha}$. Equating coefficients of $\delta t$,

(3) $\beta=\gamma-\frac{1}{2 !}(\alpha, \gamma)+\frac{1}{3 !}[\alpha,(\alpha, \gamma)]-\frac{1}{4 !}\{\alpha,[\alpha,(\alpha, \gamma)]\}+\cdots$, whence

$$
b_{k}=\sum_{1}^{r} P_{k j} c_{j} \quad(j=1,2, \cdots, r),
$$

the $P$ 's being power series in $a_{1}, \cdots, a_{r}$, convergent for all finite values of $\dot{a}_{1}, \cdots, a_{r}$.

Let $\Delta$ denote the determinant of the $P$ 's. Then if $\Delta \neq 0$ the $a$ 's and $b$ 's may be taken arbitrarily and the $c$ 's determined by means of equations (4), in which case

$$
c_{j}=\sum_{1}^{r} \frac{Q_{j k}}{d} b_{k} \quad(j=1,2, \cdots, r)
$$

where $Q_{j k}$ denotes the first minor of $\Delta$ relative to $P_{k j^{*}}$ Let the transformation $e^{a+\delta t_{\gamma}}$ be denoted by $e^{\alpha_{1}}$, where

$$
\alpha_{1}=\sum_{1}^{r} a_{k}^{(1)} X_{k}
$$

the $a_{k}^{(1)}(k=1,2, \cdots, r)$ being arbitrary parameters. Then

whence

$$
e^{a_{1}}=e^{a+\delta t \gamma}
$$

$$
a_{k}^{(1)}=a_{k}+\delta t c_{k} \quad(k=1,2, \cdots, r) .
$$


This system of equations defines the infinitesimal transformations which generate the parameter group ; but these are also defined by the equations

$$
a_{k}^{(1)}=a_{k}+\sum_{1}^{r} \xi_{k j}(a) b_{j} \delta t \quad(k=1,2, \cdots, r) .
$$

Consequently, from equations (7) and (8),

$$
c_{k}=\sum_{1}^{r} \xi_{k j}(a) b_{j}=\sum_{i}^{r} \frac{Q_{k j}}{\Delta} b_{j} .
$$

Therefore if $A_{1}, \cdots, A_{r}$ denote the symbols of the infinitesimal transformations which generate the parameter group, we have

$$
A_{j}=\sum_{1}^{r} \xi_{j k}(a) \frac{\partial}{\partial a_{k}}=\sum_{i}^{r} \frac{Q_{j k}}{\Delta} \frac{\partial}{\partial \alpha_{k}} \quad(j=1,2, \cdots, r) .
$$

Since the form of $\Delta$ and of the $Q$ 's depends only on the structural constants, the symbols $A_{j}(j=1,2, \cdots, r)$ will be the same for all groups of the same structure.

To illustrate what precedes, consider the three-parameter structure

$\left(X_{1}, X_{2}\right) \equiv 0,\left(X_{1}, X_{3}\right) \equiv X_{1},\left(X_{2}, X_{3}\right) \equiv \beta X_{2},(\beta \neq 0,1)$.

Equation (3) gives

$$
\begin{gathered}
b_{1} X_{1}+b_{2} X_{2}+b_{3} X_{3}=c_{1} X_{1}+c_{2} X_{2}+c_{3} X_{3} \\
-\frac{1}{2 !}\left\{\left(a_{1} c_{3}-a_{3} c_{1}\right) X_{1}+\beta\left(a_{2} c_{3}-a_{3} c_{2}\right) X_{2}\right\} \\
-\frac{1}{3 !}\left\{a_{3}\left(a_{1} c_{3}-a_{8} c_{1}\right) X_{1}+a_{9} \beta^{2}\left(a_{2} c_{8}-a_{3} c_{2}\right) X_{2}\right\} \\
-\frac{1}{4 !}\left\{a_{3}{ }^{2}\left(a_{1} c_{3}-a_{3} c_{1}\right) X_{1}+a_{3}{ }^{2} \beta^{3}\left(a_{2} c_{3}-a_{3} c_{2}\right) X_{2}\right\} \\
-\frac{1}{5 !}\left\{a_{3}{ }^{3}\left(a_{1} c_{3}-a_{3} c_{1}\right) X_{1}+a_{3}{ }^{3} \xi^{4}\left(a_{2} c_{3}-a_{3} c_{2}\right) X_{2}\right\}
\end{gathered}
$$

whence

$$
\begin{aligned}
& b_{1}=\frac{c_{1}}{a_{3}}\left(e^{a_{3}}-1\right)-\frac{a_{1} c_{3}}{a_{3}{ }^{2}}\left(e^{a_{3}}-a_{3}-1\right), \\
& b_{2}=\frac{c_{2}}{a_{3} \beta}\left(e^{a_{3} \beta}-1\right)-\frac{a_{2} c_{3}}{a_{3}{ }^{2} \beta}\left(e^{a_{3} \beta}-a_{3} \beta-1\right), \\
& b_{3}=c_{3} .
\end{aligned}
$$


Consequently,

$$
\Delta=\left|\begin{array}{ccc}
\frac{e^{a_{3}}-1}{a_{3}} & 0 & -\frac{a_{1}}{a_{3}^{2}}\left(e^{a_{3}}-a_{3}-1\right) \\
0 & \frac{e^{a_{3} \beta}-1}{a_{3} \beta} & -\frac{a_{2}}{a_{3}^{2} \beta}\left(e^{a_{3} \beta}-a_{3} \beta-1\right) \\
0 & 0 & 1
\end{array}\right|
$$

and equations (10) give

$$
\begin{array}{ll}
c_{1}=\frac{a_{3}}{e^{a_{3}}-1}\left\{b_{1}+\frac{a_{1}}{a_{3}^{2}}\left(e^{a_{3}}-a_{3}-1\right) b_{3}\right\} & \equiv \sum_{1}^{r} \xi_{1 j}(a) b_{j}, \\
c_{2}=\frac{a_{3} \beta}{e^{a_{3} \beta}-1}\left\{b_{2}+\frac{a_{2}}{a_{3}{ }^{2} \beta}\left(e^{a_{3} \beta}-a_{3} \beta-1\right) b_{3}\right\} & \equiv \sum_{1}^{r} \xi_{2 j}(a) b_{j}, \\
c_{3}=b_{3} & \equiv \sum_{1}^{r} \xi_{3 j}(a) b_{j} .
\end{array}
$$

Therefore the symbols of the infinitesimal transformations which generate the parameter group corresponding to the above structure are

$$
\begin{gathered}
A_{1} \equiv \frac{a_{3}}{e^{a_{3}}-1} \frac{\partial}{\partial a_{1}}, \quad A_{2} \equiv \frac{a_{3} \beta}{e^{a_{3} \beta}-1} \frac{\partial}{\partial a_{2}}, \\
A_{3} \equiv \frac{a_{1}\left(e^{a_{3}}-a_{3}-1\right)}{a_{3}\left(e^{a_{3}}-1\right)} \frac{\partial}{\partial a_{1}}+\frac{a_{2}\left(e^{a_{3} \beta}-a_{3} \beta-1\right)}{a_{3}\left(e^{a_{3} \beta}-1\right)} \frac{\partial}{\partial a_{2}}+\frac{\partial}{\partial a_{3}} .
\end{gathered}
$$

In the following table are enumerated all possible types of structure of two-, three- and four-parameter complex groups as given by Lie, $*$ and under each structure are given the symbols of the infinitesimal transformations which generate the parameter group corresponding to that structure, obtained by the method explained above.

\section{Groups With Two Parameters.}

Type $I$.

$$
\left(X_{1}, X_{2}\right) \equiv X_{1}
$$

The symbols of the infinitesimal transformations which generate the parameter group corresponding to this structure are

$$
A_{1} \equiv \frac{a_{2}}{e^{a_{2}}-1} \frac{\partial}{\partial a_{1}}, \quad A_{2} \equiv \frac{a_{1}\left(e^{a_{2}}-a_{2}-1\right)}{a_{2}\left(e^{a_{2}}-1\right)} \frac{\partial}{\partial a_{1}}+\frac{\partial}{\partial a_{2}} .
$$

* Continuierliche Gruppen, pp. 565, 571, 574-589 ; Transformationsgruppen, vol. 3, pp. 713, 716, 723-730. 
1902.] GENERATORS OF PARAMETER GROUPS.

Type II.

$$
\begin{gathered}
\left(X_{1}, X_{2}\right) \equiv 0 . \\
A_{1} \equiv \frac{\partial}{\partial \alpha_{1}}, \quad A_{2} \equiv \frac{\partial}{\partial \alpha_{2}} .
\end{gathered}
$$

Groups With Three Parameters.

Type $I . \quad\left(X_{1}, X_{2}\right) \equiv X_{1}, \quad\left(X_{1}, X_{3}\right) \equiv 2 X_{2}, \quad\left(X_{2}, X_{3}\right) \equiv X_{3}$, $A_{1} \equiv\left\{-\frac{a_{2}}{2}+\frac{\varphi\left(e^{\phi}-e^{-\phi}\right)}{2\left(e^{\phi}+e^{-\phi}-2\right)}+\frac{a_{1} a_{3}}{\varphi^{2}} \psi\right\} \frac{\partial}{\partial \alpha_{1}}$

$$
+\left\{-a_{3}+\frac{a_{2} a_{3}}{\varphi^{2}} \psi\right\} \frac{\partial}{\partial a_{2}}+\frac{a_{3}^{2}}{\varphi^{2}} \psi \frac{\partial}{\partial \alpha_{3}},
$$

$A_{2} \equiv\left\{\frac{a_{1}}{2}-\frac{a_{1} a_{2}}{2 \varphi^{2}} \psi\right\} \frac{\partial}{\partial a_{1}}+\left\{\frac{a_{2}^{2}}{\varphi^{2}}-\frac{2 a_{1} a_{3}\left(e^{\phi}-e^{-\phi}\right)}{\varphi\left(e^{\phi}+e^{-\phi}-2\right)}\right\} \frac{\partial}{\partial a_{2}}$

$A_{3} \equiv \frac{a_{1}^{2}}{\varphi^{2}} \psi^{\prime} \frac{\partial}{\partial \alpha_{1}}+\left\{a_{1}+\frac{a_{1} \alpha_{2}}{\varphi^{2}} \psi\right\} \frac{\partial}{\partial \alpha_{2}}$

$$
+\left\{-\frac{a_{3}}{2}-\frac{a_{2} a_{3}}{2 \varphi^{2}} \psi\right\} \frac{\partial}{\partial a_{3}},
$$

$$
+\left\{\frac{a_{2}}{2}+\frac{\varphi\left(e^{\phi}-e^{-\phi}\right)}{2\left(e^{\phi}+e^{-\phi}-2\right)}+\frac{a_{1} \alpha_{3}}{\varphi^{2}} \psi\right\} \frac{\partial}{\partial a_{3}},
$$

where

$$
\varphi \equiv \sqrt{a_{2}^{2}-4 a_{1} a_{3}}, \quad \psi \equiv \frac{e^{\phi}(\varphi-2)-e^{-\phi}(\varphi+2)+4}{e^{\phi}+e^{-\phi}-2} .
$$

Type II. $\left(X_{1}, X_{2}\right) \equiv 0, \quad\left(X_{1}, X_{3}\right) \equiv X_{1}, \quad\left(X_{2}, X_{3}\right) \equiv \beta X_{2}$, $(\beta \neq 0,1)$.

$$
\begin{aligned}
& A_{1} \equiv \frac{a_{3}}{e^{a_{3}}-1} \frac{\partial}{\partial a_{1}}, \quad A_{2} \equiv \frac{a_{3} \beta}{e^{a_{3} \beta}-1} \frac{\partial}{\partial a_{2}}, \\
& A_{3} \equiv \frac{a_{1}\left(e^{a_{3}}-a_{3}-1\right)}{a_{3}\left(e^{a_{3}}-1\right)} \frac{\partial}{\partial a_{1}}+\frac{a_{2}\left(a^{a_{3} \beta}-a_{3} \beta-1\right)}{a_{3}\left(e^{a_{3} \beta}-1\right)} \frac{\partial}{\partial a_{2}}+\frac{\partial}{\partial a_{3}} .
\end{aligned}
$$

Type III. $\quad\left(X_{1}, X_{2}\right) \equiv 0, \quad\left(X_{1}, X_{3}\right) \equiv X_{1}, \quad\left(X_{2}, X_{3}\right) \equiv X_{2}$

$$
\begin{aligned}
& A_{1} \equiv \frac{a_{3}}{e^{a_{3}}-1} \frac{\partial}{\partial a_{1}}, \quad A_{2} \equiv \frac{a_{3}}{e^{a_{3}}-1} \frac{\partial}{\partial \alpha_{2}}, \\
& A_{3} \equiv \frac{a_{1}\left(e^{a_{3}}-a_{3}-1\right)}{a_{3}\left(e^{a_{3}}-1\right)} \frac{\partial}{\partial a_{1}}+\frac{a_{2}\left(e^{a_{3}}-a_{3}-1\right)}{a_{3}\left(e^{a_{3}}-1\right)} \frac{\partial}{\partial a_{2}}+\frac{\partial}{\partial a_{3}} .
\end{aligned}
$$


Type IV. $\left(X_{1}, X_{2}\right) \equiv 0, \quad\left(X_{1}, X_{3}\right) \equiv X_{1}, \quad\left(X_{2}, X_{3}\right) \equiv X_{1}+X_{2}$.

$A_{1} \equiv \frac{a_{3}}{e^{a_{3}}-1} \frac{\partial}{\partial a_{1}}$

$A_{2} \equiv-\frac{a_{3}\left[e^{a_{3}}\left(a_{3}-1\right)+1\right]}{\left(e^{a_{3}}-1\right)^{2}} \frac{\partial}{\partial a_{1}}+\frac{a_{3}}{e^{a_{3}}-1} \frac{\partial}{\partial a_{2}}$,

$A_{3} \equiv\left\{\frac{a_{1}\left(e^{a_{3}}-a_{3}-1\right)+\left(a_{2}-e^{a_{2}}\right)\left[e^{a_{3}}\left(1-a_{3}\right)-1\right]}{a_{3}\left(e^{a_{3}}-1\right)}\right\} \frac{\partial}{\partial a_{1}}$

$$
+\frac{a_{2}\left(e^{a_{3}}-a_{3}-1\right)}{a_{3}\left(e^{a_{3}}-1\right)} \frac{\partial}{\partial a_{2}}+\frac{\partial}{\partial a_{3}} \text {. }
$$

Type $V . \quad\left(X_{1}, X_{2}\right) \equiv 0, \quad\left(X_{1}, X_{3}\right) \equiv X_{1}, \quad\left(X_{2}, X_{3}\right) \equiv 0$.

$$
\begin{aligned}
& A_{1} \equiv \frac{a_{3}}{e^{a_{3}}-1} \frac{\partial}{\partial a_{1}}, \quad A_{2} \equiv \frac{\partial}{\partial a_{2}}, \\
& A_{3} \equiv \frac{a_{1}\left(e^{a_{3}}-a_{3}-1\right)}{a_{3}\left(e^{a_{3}}-1\right)} \frac{\partial}{\partial a_{1}}+\frac{\partial}{\partial a_{3}} .
\end{aligned}
$$

Type VI. $\left(X_{1}, X_{2}\right) \equiv 0, \quad\left(X_{1}, X_{3}\right) \equiv 0, \quad\left(X_{2}, X_{3}\right) \equiv X_{1}$

$$
\begin{aligned}
& A_{1} \equiv \frac{\partial}{\partial a_{1}}, \quad A_{2} \equiv-\frac{a_{3}}{2} \frac{\partial}{\partial a_{1}}+\frac{\partial}{\partial a_{2}}, \\
& A_{3} \equiv \frac{a_{2}}{2} \frac{\partial}{\partial a_{1}}+\frac{\partial}{\partial a_{3}} .
\end{aligned}
$$

Type VII. $\left(X_{1}, X_{2}\right) \equiv 0, \quad\left(X_{1}, X_{3}\right) \equiv 0, \quad\left(X_{2}, X_{\mathbf{3}}\right) \equiv 0$.

$$
A_{1} \equiv \frac{\partial}{\partial a_{1}}, \quad A_{2} \equiv \frac{\partial}{\partial a_{2}}, \quad A_{3} \equiv \frac{\partial}{\partial a_{3}} \text {. }
$$

Groups With Four Parameters.

A. Without three-parameter involution group.

Type $I$.

$$
\begin{array}{lll}
\left(X_{1}, X_{2}\right) \equiv X_{1}, & \left(X_{1}, X_{3}\right) \equiv 2 X_{2}, & \left(X_{2}, X_{3}\right) \equiv X_{3}, \\
\left(X_{1}, X_{4}\right) \equiv 0, & \left(X_{2}, X_{4}\right) \equiv 0, & \left(X_{3}, X_{4}\right) \equiv 0 .
\end{array}
$$

The symbols of the infinitesimal transformations which generate the parameter group corresponding to this structure 
are the same as those given under Type $I$ of three-parameter structures with the addition of the symbol

$$
A_{4} \equiv \frac{\partial}{\partial a_{4}}
$$

Type II.

$$
\begin{aligned}
& \left(X_{1}, X_{2}\right) \equiv 0, \quad\left(X_{1}, X_{3}\right) \equiv 0, \quad\left(X_{2}, X_{3}\right) \equiv X_{1}, \\
& \left(X_{1}, X_{4}\right) \equiv \beta X_{1}, \quad\left(X_{2}, X_{4}\right) \equiv X_{2}, \quad\left(X_{3}, X_{4}\right) \equiv(\beta-1) X_{3}, \\
& A_{1} \equiv \frac{a_{4} \beta}{e^{a_{4} \beta}-1} \frac{\partial}{\partial a_{1}}, \quad(\beta \neq 1) . \\
& A_{2} \equiv \frac{a_{3}\left(\beta e^{a_{4}}-e^{a_{4} \beta}-\beta+1\right)}{\left(e^{a_{4} \beta}-1\right)\left(e^{a_{4}}-1\right)(\beta-1)} \frac{\partial}{\partial a_{1}}+\frac{a_{4}}{e^{a_{4}}-1} \frac{\partial}{\partial a_{2}}, \\
& A_{3} \equiv \frac{a_{2}\left(1-e^{a_{4} \beta}-\beta e^{-a_{4}}\right)}{\left(e^{a_{4} \beta}-1\right)\left(e^{a_{4}(\beta-1)}-1\right)} \frac{\partial}{\partial a_{1}}+\frac{a_{4}(\beta-1)}{e^{a_{4}(\beta-1)}-1} \frac{\partial}{\partial a_{3}}, \\
& A_{4} \equiv \\
& \quad \frac{1}{a_{4}\left(e^{a_{4} \beta}-1\right)}\left\{a_{1}\left(e^{a_{4} \beta}-a_{4} \beta-1\right)\right. \\
& \left.\quad+a_{2} a_{3} \beta\left[(1-\beta) e^{a_{4}(\beta-1)}+(\beta-2) e^{a_{4} \beta}+e^{a_{4}}\right]\right\} \frac{\partial}{\partial a_{1}} \\
& \quad+\frac{a_{2}\left(e^{a_{4}}-a_{4}-1\right)}{a_{4}\left(e^{a_{4}}-1\right)} \frac{\partial}{\partial a_{2}} \\
& \quad+\frac{a_{3}\left(e^{a_{4}(\beta-1)}-a_{4}(\beta-1)-1\right)}{a_{4}\left(e^{a_{4}(\beta-1)}-1\right)} \frac{\partial}{\partial a_{3}}+\frac{\partial}{\partial a_{4}} .
\end{aligned}
$$

Type III.

$$
\begin{aligned}
&\left(X_{1}, X_{2}\right) \equiv 0, \quad\left(X_{1}, X_{3}\right) \equiv 0, \quad\left(X_{2}, X_{3}\right) \equiv X_{1}, \\
&\left(X_{1}, X_{4}\right) \equiv 2 X_{1}, \quad\left(X_{2}, X_{4}\right) \equiv X_{2}, \quad\left(X_{3}, X_{4}\right) \equiv 2 X_{2}+X_{3} . \\
& A_{1} \equiv \frac{2 a_{4}}{e^{2 a_{4}}-1} \frac{\partial}{\partial a_{1}}, \\
& A_{2} \equiv-\frac{a_{3}\left(e^{2 a_{4}}-2 e^{a_{4}}+1\right)}{\left(e^{2 a_{4}}-1\right)\left(e^{a_{4}}-1\right)} \frac{\partial}{\partial a_{1}}+\frac{a_{4}}{e^{a_{4}}-1} \frac{\partial}{\partial a_{2}},
\end{aligned}
$$




$$
\begin{aligned}
& A_{3} \equiv\left\{\frac{4 a_{3}\left[\left(a_{4}-1\right) e^{a_{4}}+1\right]+a_{2}\left(e^{2 a_{4}}-2 e^{a_{4}}+1\right)}{\left(e^{2 a_{4}}-1\right)\left(e^{a_{4}}-1\right)}\right\} \frac{\partial}{\partial a_{1}} \\
&-\frac{2 a_{4}\left(a_{4} e^{a_{4}}-e^{a_{4}}+1\right)}{\left(e^{a_{4}}-1\right)^{2}} \frac{\partial}{\partial a_{2}}+\frac{a_{4}}{e^{a_{4}}-1} \frac{\partial}{\partial a_{3}}, \\
& A_{4} \equiv\left\{e^{a_{4}}\left[\left(2-a_{4}\right)\left(e^{2 a_{4}}+1\right)+2\left(a_{4}-2 e^{a_{4}}\right)\right]\right\} \frac{\partial}{\partial a_{1}} \\
&+\frac{2 a_{3}\left(a_{4} e^{a_{4}}-e^{a_{4}}+1\right)}{e^{a_{4}}-1} \frac{\partial}{\partial a_{2}}+\frac{a_{3}\left(e^{a_{4}}-a_{4}-1\right)}{a_{4}\left(e^{a_{4}}-1\right)} \frac{\partial}{\partial a_{3}}+\frac{\partial}{\partial a_{4}} .
\end{aligned}
$$

Type IV.

$$
\begin{array}{r}
\left(X_{1}, X_{2}\right) \equiv 0, \quad\left(X_{1}, X_{3}\right) \equiv 0, \quad\left(X_{2}, X_{3}\right) \equiv X_{1}, \\
\left(X_{1}, X_{4}\right) \equiv X_{1}, \quad\left(X_{2}, X_{4}\right) \equiv X_{2}, \quad\left(X_{3}, X_{4}\right) \equiv 0 . \\
A_{1} \equiv \frac{a_{4}}{e^{a_{4}}-1} \frac{\partial}{\partial a_{1}}, \\
A_{2} \equiv-\frac{a_{3}\left[e^{a_{4}}\left(a_{4}-1\right)+\frac{a_{4}^{2}}{2}+1\right]}{\left(e^{a_{4}}-1\right)^{2}} \frac{\partial}{\partial a_{1}}+\frac{a_{4}}{e^{a_{4}}-1} \frac{\partial}{\partial a_{2}}, \\
A_{8} \equiv \frac{a_{2}\left(e^{a_{4}}-a_{4}-1\right)}{a_{4}\left(e^{a_{4}}-1\right)} \frac{\partial}{\partial a_{1}}+\frac{\partial}{\partial a_{3}}, \\
A_{4} \equiv\left\{\frac{a_{1}\left(e^{a_{4}}-a_{4}-1\right)}{a_{4}\left(e^{a_{4}}-1\right)}+\frac{a_{2} a_{3}}{e^{a_{4}}-1}\left[e^{a_{4}}\left(a_{4}-3\right)\left(e^{a_{4}}+\frac{a_{4}}{2}\right)\right.\right. \\
\left.\left.+\frac{a_{4}+2}{2}\left(4 e^{a_{4}}-1\right)\right]\right\} \frac{\partial}{\partial a_{1}}+\frac{a_{2}\left(e^{a_{4}}-a_{4}-1\right)}{a_{4}\left(e^{a_{4}}-1\right)} \frac{\partial}{\partial a_{2}}+\frac{\partial}{\partial a_{4}} .
\end{array}
$$

Type V.

$$
\begin{aligned}
\left(X_{1}, X_{2}\right) & \equiv 0, \quad\left(X_{1}, X_{3}\right) \equiv 0, \quad\left(X_{2}, X_{3}\right) \equiv X_{2} \\
\left(X_{1}, X_{4}\right) & \equiv X_{1}, \quad\left(X_{2}, X_{4}\right) \equiv 0, \quad\left(X_{3}, X_{4}\right) \equiv 0 . \\
A_{1} & \equiv \frac{a_{4}}{e^{a_{4}}-1} \frac{\partial}{\partial a_{1}}, \quad A_{2} \equiv \frac{a_{3}}{e^{a_{3}}-1} \frac{\partial}{\partial \alpha_{2}}, \\
A_{3} & \equiv \frac{a_{2}\left(e^{a_{3}}-a_{3}-1\right)}{a_{3}\left(e^{a_{3}}-1\right)} \frac{\partial}{\partial a_{2}}+\frac{\partial}{\partial a_{3}}, \\
A_{4} & \equiv \frac{a_{1}\left(e^{a_{4}}-a_{4}-1\right)}{a_{4}\left(e^{a_{4}}-1\right)} \frac{\partial}{\partial a_{1}}+\frac{\partial}{\partial a_{4}} .
\end{aligned}
$$


1902.] GENERATORS OF PARAMETER GROUPS.

B. With three-parameter involution group.

Type $I$.

$$
\begin{gathered}
\left(X_{1}, X_{2}\right) \equiv\left(X_{2}, X_{3}\right) \equiv\left(X_{3}, X_{1}\right) \equiv 0, \\
\left(X_{1}, X_{4}\right) \equiv a X_{1}, \quad\left(X_{2}, X_{4}\right) \equiv \beta X_{2}, \quad\left(X_{3}, X_{4}\right) \equiv \gamma X_{3}, \\
(\alpha \neq \beta \neq \gamma) . \\
A_{1} \equiv \frac{a_{4} \alpha}{e^{a_{4} \alpha}-1} \frac{\partial}{\partial a_{1}}, \quad A_{2} \equiv \frac{a_{4^{\prime}}}{e^{a_{4} \beta}-1} \frac{\partial}{\partial a_{2}}, \quad A_{3} \equiv \frac{a_{4} \gamma}{e^{a_{4} \gamma}-1} \frac{\partial}{\partial a_{8}}, \\
A_{4} \equiv \frac{a_{1}\left(e^{a_{4} a}-a_{4} \alpha-1\right)}{a_{4}\left(e^{a_{4} \alpha}-1\right)} \frac{\partial}{\partial a_{1}}+\frac{a_{2}\left(e^{a_{4} \beta}-a_{4} \beta-1\right)}{a_{4}\left(e^{a_{4} \beta}-1\right)} \frac{\partial}{\partial a_{2}} \\
\quad+\frac{a_{3}\left(e^{a_{4} \gamma}-a_{4} \gamma-1\right)}{a_{4}\left(e^{a_{4} \gamma}-1\right)} \frac{\partial}{\partial a_{3}}+\frac{\partial}{\partial a_{4}} .
\end{gathered}
$$

Type II.

$$
\begin{aligned}
&\left(X_{1}, X_{2}\right) \equiv\left(X_{2}, X_{3}\right) \equiv\left(X_{3}, X_{1}\right) \equiv 0, \\
&\left(X_{1}, X_{4}\right) \equiv \alpha X_{1}, \quad\left(X_{2}, X_{4}\right) \equiv \beta X_{2}, \quad\left(X_{3}, X_{4}\right) \equiv X_{2}+\beta X_{3},(\alpha \neq \beta) . \\
& A_{1} \equiv \frac{a_{4} \alpha}{e^{a_{4} a}-1} \frac{\partial}{\partial a_{1}}, \quad A_{2} \equiv \frac{a_{4} \beta}{e^{a_{4} \beta}-1} \frac{\partial}{\partial a_{2}}, \\
& A_{3} \equiv \frac{a_{4}\left[e^{a_{4} \beta}\left(a_{4} \beta-1\right)+1\right]}{\left(e^{a_{4} \beta}-1\right)^{2}} \frac{\partial}{\partial a_{2}}+\frac{a_{4} \beta}{e^{a_{4} \beta}-1} \frac{\partial}{\partial a_{3}}, \\
& A_{4} \equiv \frac{a_{1}\left(e^{a_{4} a}-a_{4} \alpha-1\right)}{a_{4}\left(e^{a_{4} a}-1\right)} \frac{\partial}{\partial a_{1}} \\
&+\frac{1}{a_{4} \beta\left(e^{a_{4} \beta}-1\right)}\left\{a_{3}\left[e^{a_{4} \beta}\left(a_{4} \beta-1\right)+1\right]\left(2 e^{a_{4} \beta}-a_{4} \beta-2\right)\right. \\
&\left.+a_{2} \beta\left(e^{a_{4} \beta}-a_{4} \beta-1\right)\right\} \frac{\partial}{\partial a_{2}}+\frac{a_{3}\left(e^{a_{4} \beta}-a_{4} \beta-1\right)}{a_{4}\left(e^{a_{4} \beta}-1\right)} \frac{\partial}{\partial a_{3}}+\frac{\partial}{\partial a_{4}} .
\end{aligned}
$$

Type III.

$$
\begin{aligned}
& \left(X_{1}, X_{2}\right) \equiv\left(X_{2}, X_{3}\right) \equiv\left(X_{3}, X_{1}\right) \equiv 0, \\
& \left(X_{1}, X_{4}\right) \equiv X_{1}, \quad\left(X_{2}, X_{4}\right) \equiv X_{1}+X_{2}, \quad\left(X_{3}, X_{4}\right) \equiv X_{2}+X_{3} . \\
& A_{1} \equiv \frac{a_{4}}{e^{a_{4}}-1} \frac{\partial}{\partial a_{1}}, \\
& A_{2} \equiv \frac{a_{4}\left[e^{a_{4}}\left(a_{4}-1\right)+1\right]}{\left(e^{a_{4}}-1\right)^{2}} \frac{\partial}{\partial a_{1}}+\frac{a_{4}}{e^{a_{4}}-1} \frac{\partial}{\partial a_{2}},
\end{aligned}
$$




$$
\begin{gathered}
A_{3} \equiv \frac{a_{4}^{2} e^{a_{4}}}{\left(e^{a_{4}}-1\right)^{3}}\left[e^{a_{4}}-\frac{\alpha_{4}}{2}\left(e^{a_{4}}+1\right)-1\right] \frac{\partial}{\partial a_{1}} \\
\quad-\frac{a_{4}\left[e^{a_{4}}\left(a_{4}-1\right)+1\right]}{\left(e^{a_{4}}-1\right)^{2}} \frac{\partial}{\partial a_{2}}+\frac{a_{4}}{e^{a_{4}}-1} \frac{\partial}{\partial a_{3}}, \\
A_{4} \equiv \frac{1}{a_{4}\left(e^{a_{4}}-1\right)}\left\{a_{1}\left(e^{a_{4}}-a_{4}-1\right)\right. \\
+\frac{a_{2}\left(a_{4} e^{a_{4}}-e^{a_{4}}+1\right)\left(2 e^{a_{4}}-a_{4}-2\right)}{2}\left[\left(a_{4}^{2} e^{a_{4}}-2 a_{4} e^{a_{4}}+2 e^{a_{4}}-2\right) \frac{2 e^{a_{4}}-a_{4}-2}{2}\right. \\
\left.\left.+\frac{a_{3}\left(a_{4} e^{a_{4}}-e^{a_{4}}+1\right)^{2}}{e^{a_{4}}-1}\right]\right\} \frac{\partial}{\partial a_{1}} \\
+\frac{1}{a_{4}\left(e^{a_{4}}-1\right)}\left\{a_{2}\left(e^{a_{4}}-a_{4}-1\right)+a_{3} a_{4}\left(a_{4} e^{a_{4}}-e^{a_{4}}+1\right)\right\} \frac{\partial}{\partial a_{2}} \\
\quad+\frac{a_{3}\left(e^{a_{4}}-a_{4}-1\right)}{a_{4}\left(e^{a_{4}}-1\right)} \frac{\partial}{\partial a_{3}}+\frac{\partial}{\partial a_{4}} .
\end{gathered}
$$

Type IIIa.

$$
\begin{aligned}
\left(X_{1}, X_{2}\right) & \equiv\left(X_{2}, X_{3}\right) \equiv\left(X_{3}, X_{1}\right) \equiv 0, \\
\left(X_{1}, X_{4}\right) & \equiv 0, \quad\left(X_{2}, X_{4}\right) \equiv X_{1}, \quad\left(X_{3}, X_{4}\right) \equiv X_{2} . \\
A_{1} & \equiv \frac{\partial}{\partial a_{1}}, \quad A_{2} \equiv-\frac{a_{4}}{2} \frac{\partial}{\partial a_{1}}+\frac{\partial}{\partial a_{2}}, \\
A_{3} & \equiv \frac{a_{4}^{2}}{12} \frac{\partial}{\partial a_{1}}-\frac{a_{4}}{2} \frac{\partial}{\partial \alpha_{2}}+\frac{\partial}{\partial a_{3}}, \\
A_{4} & \equiv \frac{1}{2}\left(a_{2}-\frac{a_{3} a_{4}}{6}\right) \frac{\partial}{\partial a_{1}}+\frac{a_{3}}{2} \frac{\partial}{\partial a_{2}}+\frac{\partial}{\partial a_{4}} .
\end{aligned}
$$

Type $I V$.

$$
\begin{gathered}
\left(X_{1}, X_{2}\right) \equiv\left(X_{2}, X_{3}\right) \equiv\left(X_{3}, X_{1}\right) \equiv 0, \\
\left(X_{1}, X_{4}\right) \equiv \alpha X_{1}, \quad\left(X_{2}, X_{4}\right) \equiv \alpha X_{2}, \quad\left(X_{3}, X_{4}\right) \equiv \gamma X_{3}, \\
(\alpha \neq \gamma) . \\
A_{1} \equiv \frac{a_{4}^{\alpha}}{e^{a_{4} \alpha}-1} \frac{\partial}{\partial \alpha_{1}}, \quad A_{2} \equiv \frac{a_{4} \alpha}{e^{a_{4} \alpha}-1} \frac{\partial}{\partial \alpha_{2}}, \quad A_{3} \equiv \frac{a_{4} \gamma}{e^{a_{4} \gamma}-1} \frac{\partial}{\partial \alpha_{3}},
\end{gathered}
$$


1902.] GENERATORS OF PARAMETER GROUPS.

$A_{4} \equiv \frac{a_{1}\left(e^{a_{4} a}-a_{4} \alpha-1\right)}{a_{4}\left(e^{a_{4} \alpha}-1\right)} \frac{\partial}{\partial a_{1}}+\frac{a_{2}\left(e^{a_{4} \alpha}-a_{4} \alpha-1\right)}{a_{4}\left(e^{a_{4} a}-1\right)} \frac{\partial}{\partial a_{2}}$

Type $V$.

$$
+\frac{a_{3}\left(e^{a_{4} \gamma}-a_{4} \gamma-1\right)}{a_{4}\left(e^{a_{4} \gamma}-1\right)} \frac{\partial}{\partial a_{3}}+\frac{\partial}{\partial a_{4}} \text {. }
$$

$$
\begin{aligned}
&\left(X_{1}, X_{2}\right) \equiv\left(X_{2}, X_{3}\right) \equiv\left(X_{3}, X_{1}\right) \equiv 0, \\
&\left(X_{1}, X_{4}\right) \equiv X_{1}, \quad\left(X_{2}, X_{4}\right) \equiv X_{2}, \quad\left(X_{3}, X_{4}\right) \equiv X_{2}+X_{3} . \\
& A_{1} \equiv \frac{a_{4}}{e^{a_{4}}-1} \frac{\partial}{\partial a_{1}}, \quad A_{2} \equiv \frac{a_{4}}{e^{a_{4}}-1} \frac{\partial}{\partial a_{2}}, \\
& A_{3} \equiv-\frac{a_{4}\left[e^{a_{4}}\left(a_{4}-1\right)+1\right]}{\left(e^{a_{4}}-1\right)^{2}} \frac{\partial}{\partial a_{2}}+\frac{a_{4}}{e^{a_{4}}-1} \frac{\partial}{\partial a_{3}}, \\
& A_{4} \equiv \frac{a_{1}\left(e^{a_{4}}-a_{4}-1\right)}{a_{4}\left(e^{a_{4}}-1\right)} \frac{\partial}{\partial a_{1}}+\frac{1}{a_{4}\left(e^{a_{4}}-1\right)}\left\{a_{2}\left(e^{a_{4}}-a_{4}-1\right)\right. \\
&\left.\quad+a_{3} a_{4}\left(a_{4} e^{a_{4}}-e^{a_{4}}+1\right)\right\} \frac{\partial}{\partial a_{2}}+\frac{a_{3}\left(e^{a_{4}}-a_{4}-1\right)}{a_{4}\left(e^{a_{4}}-1\right)} \frac{\partial}{\partial a_{3}}+\frac{\partial}{\partial a_{4}} .
\end{aligned}
$$

Type Va.

$$
\begin{aligned}
& \left(X_{1}, X_{2}\right) \equiv\left(X_{2}, X_{3}\right) \equiv\left(X_{3}, X_{1}\right) \equiv 0, \\
& \left(X_{1}, X_{4}\right) \equiv 0, \quad\left(X_{2}, X_{4}\right) \equiv 0, \quad\left(X_{3}, X_{4}\right) \equiv X_{2} . \\
& A_{1} \equiv \frac{\partial}{\partial a_{1}}, \quad A_{2} \equiv \frac{\partial}{\partial a_{2}}, \\
& A_{3} \equiv-\frac{a_{4}}{2} \frac{\partial}{\partial a_{2}}+\frac{\partial}{\partial a_{3}}, \quad A_{4} \equiv \frac{\alpha_{3}}{2} \frac{\partial}{\partial a_{2}}+\frac{\partial}{\partial a_{4}}
\end{aligned}
$$

Type VI.

$$
\begin{gathered}
\left(X_{1}, X_{2}\right) \equiv\left(X_{2}, X_{3}\right) \equiv\left(X_{3}, X_{1}\right) \equiv 0, \\
\left(X_{1}, X_{4}\right) \equiv X_{1}, \quad\left(X_{2}, X_{4}\right) \equiv X_{2}, \quad\left(X_{3}, X_{4}\right) \equiv X_{3} . \\
A_{1} \equiv \frac{a_{4}}{e^{a_{4}}-1} \frac{\partial}{\partial a_{1}}, \quad A_{2} \equiv \frac{a_{4}}{e^{a_{4}}-1} \frac{\partial}{\partial a_{2}}, \quad A_{3} \equiv \frac{a_{4}}{e^{a_{4}}-1} \frac{\partial}{\partial a_{3}}, \\
A_{4} \equiv \frac{a_{1}\left(e^{a_{4}}-a_{4}-1\right)}{a_{4}\left(e^{a_{4}}-1\right)} \frac{\partial}{\partial a_{1}}+\frac{a_{2}\left(e^{a_{4}}-a_{4}-1\right)}{a_{4}\left(e^{a_{4}}-1\right)} \frac{\partial}{\partial a_{2}} \\
\quad+\frac{a_{3}\left(e^{a_{4}}-a_{4}-1\right)}{a_{4}\left(e^{a_{4}}-1\right)} \frac{\partial}{\partial a_{3}}+\frac{\partial}{\partial a_{4}} .
\end{gathered}
$$


Type VIa.

$$
\begin{gathered}
\left(X_{1}, X_{2}\right) \equiv\left(X_{2}, X_{3}\right) \equiv\left(X_{3}, X_{1}\right) \equiv 0, \\
\left(X_{1}, X_{4}\right) \equiv 0, \quad\left(X_{2}, X_{4}\right) \equiv 0, \quad\left(X_{3}, X_{4}\right) \equiv 0 . \\
A_{1} \equiv \frac{\partial}{\partial a_{1}}, \quad A_{2} \equiv \frac{\partial}{\partial a_{2}}, \quad A_{3} \equiv \frac{\partial}{\partial a_{3}}, \quad A_{4} \equiv \frac{\partial}{\partial a_{4}} .
\end{gathered}
$$

UNIVERSity OF CINCINNATI,

October, 1901.

\section{SHORTER NOTICES.}

Einführung in die Theorie der Differentialgleichungen mit einer unabhängigen Variablen. Von Dr. Ludwig Schlesinger, ordentlichem Professor an der Universität zu Klausenburg. Leipzig, Göschen, 1900. Pp. viii +310 .

THIs little volume, which forms part of the "Sammlung Schubert". (cf. the Bulletin for January, 1901, p. 192), gives, we believe, the best introduction which has yet appeared to that important side of the theory of ordinary differential equations in which the points of view are those of the theory of functions of a complex variable. Thus the discussion of the nature of singular points holds a central position in the treatment given. The author has been particularly successful in his choice of topics. He has on the one hand restricted himself to the simpler parts of the subject, more than half the volume being devoted to linear differential equations of the second order, and the remainder to the case of a single equation of the first order. By doing this he has succeeded in avoiding long analytical developments which only confuse a beginner without really teaching him anything. On the other hand the author has treated these simple cases in such a way as to bring out clearly a large number of the most important points of view of the modern theory of differential equations. Some of Dr. Schlesinger's own investigations, to mention only one point, on the Laplacian and Eulerian transformations are here set forth in particularly attractive form, although, of course, only for very special differential equations. The reader can turn to a large treatise for further information on these or 